\title{
Some examples of cocycles with simple continuous singular spectrum
}

\author{
by
}

KRZYSZTOF FRĄCZEK (Toruń)

\begin{abstract}
We study spectral properties of Anzai skew products $T_{\varphi}: \mathbb{T}^{2} \rightarrow \mathbb{T}^{2}$ defined by

$$
T_{\varphi}(z, \omega)=\left(e^{2 \pi i \alpha} z, \varphi(z) \omega\right),
$$

where $\alpha$ is irrational and $\varphi: \mathbb{T} \rightarrow \mathbb{T}$ is a measurable cocycle. Precisely, we deal with the case where $\varphi$ is piecewise absolutely continuous such that the sum of all jumps of $\varphi$ equals zero. It is shown that the simple continuous singular spectrum of $T_{\varphi}$ on the orthocomplement of the space of functions depending only on the first variable is a "typical" property in the above-mentioned class of cocycles, if $\alpha$ admits a sufficiently fast approximation.
\end{abstract}

1. Introduction. By $\mathbb{T}$ we denote the circle group $\{z \in \mathbb{C}:|z|=1\}$ which will most often be treated as the interval $[0,1)$ with addition mod 1 ; $\lambda$ will denote Lebesgue measure on $\mathbb{T}$. A function $f: \mathbb{T} \rightarrow \mathbb{R}$ is said to be piecewise absolutely continuous (PAC for short) if there exist $\beta_{0}, \ldots, \beta_{k} \in \mathbb{T}$ $\left(0 \leq \beta_{0}<\ldots<\beta_{k}<1\right)$ such that $\left.f\right|_{\left(\beta_{j}, \beta_{j+1}\right)}$ is absolutely continuous $\left(\beta_{k+1}=\beta_{0}\right)$. Then we set

$$
f_{+}(x)=\lim _{y \rightarrow x^{+}} f(y) \quad \text { and } \quad f_{-}(x)=\lim _{y \rightarrow x^{-}} f(y) .
$$

Let $d_{j}=f_{+}\left(\beta_{j}\right)-f_{-}\left(\beta_{j}\right)$ for $j=0, \ldots, k$ and

$$
S(f)=\sum_{j=0}^{k} d_{j}=-\sum_{j=0}^{k}\left(f_{-}\left(\beta_{j}\right)-f_{+}\left(\beta_{j}\right)\right)=-\int_{\mathbb{T}} D f(x) d \lambda(x) .
$$

We call a function $\varphi: \mathbb{T} \rightarrow \mathbb{T}$ piecewise absolutely continuous if there exists a PAC function $\widetilde{\varphi}: \mathbb{T} \rightarrow \mathbb{R}$ such that $\varphi\left(e^{2 \pi i x}\right)=e^{2 \pi i \widetilde{\varphi}(x)}$. Set $S(\varphi)=S(\widetilde{\varphi})$. Since the number $S(\widetilde{\varphi})$ is independent of the choice of the function $\widetilde{\varphi}$, the number $S(\varphi)$ is well defined and will be called the sum of jumps of $\varphi$.

2000 Mathematics Subject Classification: Primary 37A05.

Research partly supported by KBN grant 2 P03A 002 14(1998), by FWF grant P12250-MAT and by Foundation for Polish Science. 
Let $\alpha \in \mathbb{T}$ be irrational. Denote by $T z=e^{2 \pi i \alpha} z(T x=x+\alpha \bmod 1)$ the corresponding ergodic rotation on $\mathbb{T}$. We will study spectral properties of measure preserving automorphisms of $\mathbb{T}^{2}$ (called Anzai skew products) defined by

$$
T_{\varphi}(z, \omega)=(T z, \varphi(z) \omega)
$$

where $\varphi: \mathbb{T} \rightarrow \mathbb{T}$ is a PAC function.

Consider the Koopman unitary operator $U_{T_{\varphi}}: L^{2}(\mathbb{T} \times \mathbb{T}, \lambda \otimes \lambda) \rightarrow$ $L^{2}(\mathbb{T} \times \mathbb{T}, \lambda \otimes \lambda)$ associated with the Anzai skew product $T_{\varphi}$ and defined by $U_{T_{\varphi}}=f \circ T_{\varphi}$. Let us decompose

$$
L^{2}(\mathbb{T} \times \mathbb{T}, \lambda \otimes \lambda)=\bigoplus_{m \in \mathbb{Z}} H_{m}
$$

where

$$
H_{m}=\left\{g: g(z, \omega)=f(z) \omega^{m}, f \in L^{2}(\mathbb{T}, \lambda)\right\} .
$$

Observe that $H_{m}$ is a closed $U_{T_{\varphi}}$-invariant subspace of $L^{2}(\mathbb{T} \times \mathbb{T}, \lambda \otimes \lambda)$. Moreover the operator $U_{T_{\varphi}}: H_{m} \rightarrow H_{m}$ is unitarily equivalent to the operator $U_{\varphi}^{(m)}: L^{2}(\mathbb{T}, \lambda) \rightarrow L^{2}(\mathbb{T}, \lambda)$ given by

$$
\left(U_{\varphi}^{(m)} f\right)(z)=\varphi(z)^{m} f(T z) .
$$

This leads to the problem of spectral classification of unitary operators $V_{g}$ : $L^{2}(\mathbb{T}, \lambda) \rightarrow L^{2}(\mathbb{T}, \lambda)$ given by $V_{g} f(z)=g(z) f(T z)$, where $g: \mathbb{T} \rightarrow \mathbb{T}$ is a measurable function.

Let $U$ be a unitary operator on a separable Hilbert space $\mathcal{H}$. For any $f \in \mathcal{H}$ we define the cyclic space $\mathbb{Z}(f)=\operatorname{span}\left\{U^{n} f: n \in \mathbb{Z}\right\}$. By the spectral measure $\sigma_{f}$ of $f$ we mean a Borel measure on $\mathbb{T}$ determined by the equalities

$$
\widehat{\sigma}_{f}(n)=\int_{\mathbb{T}} z^{n} d \sigma_{f}(z)=\left(U^{n} f, f\right)
$$

for $n \in \mathbb{Z}$. Let $\left\{f_{n}\right\}_{n \in \mathbb{N}}$ be a sequence in $\mathcal{H}$ such that

$$
\mathcal{H}=\bigoplus_{n=1}^{\infty} \mathbb{Z}\left(f_{n}\right) \quad \text { and } \quad \sigma_{f_{1}} \gg \sigma_{f_{2}} \gg \ldots
$$

The spectral type of $\sigma_{f_{1}}$ (the equivalence class of measures) will be called the maximal spectral type of $U$. We say that $U$ has Lebesgue (resp. continuous singular, discrete) spectrum if $\sigma_{f_{1}}$ is equivalent to Lebesgue (resp. continuous singular, discrete) measure on the circle. A number $m \in \mathbb{N} \cup\{\infty\}$ is called the maximal spectral multiplicity of $U$ if $\sigma_{f_{n}} \not \equiv 0$ for $n \leq m$ and $\sigma_{f_{n}} \equiv 0$ for $n>m$. We say that $U$ has simple spectrum if the maximal spectral multiplicity of $U$ equals 1 .

The notion of the skew product was introduced in 1951 by Anzai (see [1]) to give some examples of ergodic transformations with some special spectral types. Anzai skew products or more generally operators $V_{g}$ have a 
well known property called the purity law. Precisely, each operator $V_{g}$ has either Lebesgue or continuous singular or discrete spectrum (see [6] and [10]).

In the case where $\varphi: \mathbb{T} \rightarrow \mathbb{T}$ is a smooth cocycle, the spectral properties of $T_{\varphi}$ depend on the value of the topological degree of $\varphi$, which equals $-S(\varphi)$. For example, if $\varphi$ is of class $C^{2}$ and $S(\varphi) \neq 0$, then $T_{\varphi}$ has countable Lebesgue spectrum on $H_{0}^{\perp}$ (see [2] and [10]). On the other hand, $S(\varphi)=0$ implies singular spectrum for absolutely continuous $\varphi$ (see [3]). In this case, numerous dynamical properties of the skew product depend on properties of the continued fraction expansion of $\alpha$. For example, each smooth cocycle with zero degree is cohomologous to a constant if $\alpha$ admits a sufficiently slow approximation. It follows that the skew product has pure discrete spectrum. On the other hand, if $\alpha$ admits a sufficiently fast approximation, then the skew product associated with a generic $C^{r}$-cocycle $(r \in \mathbb{N} \cup\{\infty\})$ with zero degree has simple continuous singular spectrum of $T_{\varphi}$ on $H_{0}^{\perp}$ (see [8]). Generally, we also have some information about multiplicity of $U_{\varphi}^{(m)}$. For every absolutely continuous $g: \mathbb{T} \rightarrow \mathbb{T}$, the multiplicity of $V_{g}$ is at most $\max (1,|S(g)|)($ see $[5])$.

In the case where $\varphi: \mathbb{T} \rightarrow \mathbb{T}$ is PAC, the spectral properties of $T_{\varphi}$ also depend on the value $S(\varphi)$. For example, $S(\varphi) \neq 0$ implies continuous spectrum on $H_{0}^{\perp}$ (see [9]). Moreover, if $\varphi$ has a single discontinuity with $S(\varphi) \in \mathbb{R} \backslash \mathbb{Z}$, then $T_{\varphi}$ has continuous singular spectrum on $H_{0}^{\perp}$.

In the paper we deal with the case where $S(\varphi)=0$. Generally, it is shown in [5] that the multiplicity of each operator $U_{\varphi}^{(m)}$ is at most the number of discontinuities of $\varphi$. However, every piecewise constant cocycle such that all the discontinuities of $\varphi$ are multiples of $\alpha$ is cohomologous to a constant cocycle, because each cocycle of the form $\varphi\left(e^{2 \pi i x}\right)=e^{2 \pi i a \mathbf{1}_{[0, k \alpha)}(x)+b}, k \in \mathbb{Z}$, is cohomologous to a constant cocycle (see [7], p. 82). Then $T_{\varphi}$ has discrete spectrum. If $\varphi$ has only rational jumps (i.e. $d_{0}, \ldots, d_{k} \in \mathbb{Q}$ ), then $\varphi^{m}$ is constant for a nonzero $m$, hence $U_{\varphi}^{(m)}$ also has discrete spectrum. On the other hand, we will show that the simple continuous singular spectrum of $T_{\varphi}$ on $H_{0}^{\perp}$ is a "typical" property for PAC cocycles whose sum of jumps equals zero, if $\alpha$ admits a sufficiently fast approximation.

For every natural $k$ define

$$
\mathbb{T}_{+}^{k}=\left\{\left(x_{1}, \ldots, x_{k}\right) \in \mathbb{T}^{k}: 0 \leq x_{1}<\ldots<x_{k}<1\right\} .
$$

We will prove the following assertion.

Theorem 1.1 [Main Theorem]. Let $\alpha \in \mathbb{T}$ be an irrational number with unbounded partial quotients in its continued fraction expansion. For every $k \in \mathbb{N}$, there exists a subset $B_{k+1} \subset \mathbb{T}_{+}^{k+1}$ of full Lebesgue measure such that if $\varphi: \mathbb{T} \rightarrow \mathbb{T}$ is a PAC function with 
- $S(\varphi)=0$

- at least one of its jumps being irrational;

- $k+1$ discontinuities $\beta_{0}, \ldots, \beta_{k}$ satisfying $\left(\beta_{0}, \ldots, \beta_{k}\right) \in B_{k+1}$, then $T_{\varphi}$ has simple continuous singular spectrum on $H_{0}^{\perp}$.

To prove this theorem we will use the idea of $\delta$-weak mixing. Let $\delta$ be a complex number such that $|\delta| \leq 1$. We say that a unitary operator $U: \mathcal{H} \rightarrow \mathcal{H}$ is $\delta$-weakly mixing along a sequence $\left\{q_{n}\right\}_{n \in \mathbb{N}}$ if

$$
\lim _{n \rightarrow \infty}\left(U^{q_{n}} f, f\right)=\delta(f, f)
$$

for any $f \in \mathcal{H}$.

A simple spectral analysis gives the following well known fact.

Proposition 1. Let $U_{i}: \mathcal{H}_{i} \rightarrow \mathcal{H}_{i}, i=1,2$, be a unitary operator on a separable Hilbert space. Assume that the $U_{i}$ are $\delta_{i}$-weakly mixing along a common sequence $\left\{q_{n}\right\}_{n \in \mathbb{N}}$. If $\delta_{1} \neq \delta_{2}$, then the maximal spectral types of $U_{i}$ are mutually singular.

We will apply the concept of the $\delta$-weak mixing to the family of unitary operators $\left(U_{\varphi}^{(m)}\right)$. We say that an increasing sequence $\left\{q_{n}\right\}_{n \in \mathbb{N}}$ of natural numbers is a rigid time for $T$ if

$$
\lim _{n \rightarrow \infty}\left\|q_{n} \alpha\right\|=0
$$

where $\|t\|$ is the distance of $t$ from the set of integers. For given $\varphi: \mathbb{T} \rightarrow \mathbb{T}$ and $q \in \mathbb{N}$ let

$$
\varphi^{(q)}(z)=\varphi(z) \varphi(T z) \ldots \varphi\left(T^{q-1} z\right) .
$$

Proposition 2 (see [4]). Assume that

$$
\lim _{n \rightarrow \infty} \int_{\mathbb{T}}\left(\varphi^{\left(q_{n}\right)}(z)\right)^{m} d z=\delta_{m}
$$

where $\left\{q_{n}\right\}_{n \in \mathbb{N}}$ is a rigid time for $T$. Then the operator $U_{\varphi}^{(m)}$ is $\delta_{m}$-weakly mixing along $\left\{q_{n}\right\}_{n \in \mathbb{N}}$.

2. The definition of the set $B_{k}$. Assume that $\alpha \in[0,1)$ is irrational with continued fraction expansion

$$
\alpha=\left[0 ; a_{1}, a_{2}, \ldots\right] .
$$

Let $\left(p_{n} / q_{n}\right)$ be the convergents of $\alpha$; then

$$
\left\|\alpha q_{n}\right\|=\left|q_{n} \alpha-p_{n}\right|<1 / q_{n+1}
$$

and $\left(T^{j}\left[0,\left\|\alpha q_{n-1}\right\|\right)\right)_{0 \leq j<q_{n}}$ is a tower (i.e. a family of pairwise disjoint sets). We also have

$$
\left\|\alpha q_{n+1}\right\|=a_{n+1}\left\|\alpha q_{n}\right\|+\left\|\alpha q_{n-1}\right\| \quad \text { and } \quad\left\|\alpha q_{n-1}\right\| q_{n}+\left\|\alpha q_{n}\right\| q_{n-1}=1 .
$$


We shall consider $\alpha$ with unbounded partial quotients, i.e. we can choose a subsequence, still denoted by $n$, such that $\lim _{n \rightarrow \infty} a_{n+1}=\infty$. Then, with the previous relations, $q_{n}\left\|q_{n} \alpha\right\| \rightarrow 0$ and $q_{n}\left\|q_{n-1} \alpha\right\| \rightarrow 1$.

Lemma 2.1. Let $0<\tau<1$ and let $W=\prod_{i=1}^{k}\left[v_{i}, w_{i}\right]$ be a closed cube in $\mathbb{T}^{k}$ with $\lambda^{k}(W)>0$. For almost every $\left(\beta_{1}, \ldots, \beta_{k}\right) \in \mathbb{T}^{k}$ there exists a subsequence $\left\{q_{n_{j}}\right\}_{j \in \mathbb{N}}$ such that

$$
\lim _{j \rightarrow \infty} q_{n_{j}}\left\|q_{n_{j}} \alpha\right\|=0, \quad \lim _{j \rightarrow \infty}\left(\left\{q_{n_{j}} \beta_{1}\right\}, \ldots,\left\{q_{n_{j}} \beta_{k}\right\}\right)=\left(\gamma_{1}, \ldots, \gamma_{k}\right) \in W
$$

and

$$
\beta_{1}, \ldots, \beta_{k} \in \bigcup_{\tau q_{n_{j}}<t<q_{n_{j}}} T^{t}\left[0,\left\|q_{n_{j}-1} \alpha\right\|\right)
$$

for every natural $j$.

Proof. Assume that $\left\{\Xi_{n}\right\}_{n \in \mathbb{N}}$ is a sequence of towers for the rotation $T$ for which $\liminf \operatorname{in}_{n \rightarrow \infty} \lambda\left(\Xi_{n}\right)>0$ and height $\left(\Xi_{n}\right) \rightarrow \infty$. Then

$$
\lambda\left(B \cap \Xi_{n}\right)-\lambda(B) \lambda\left(\Xi_{n}\right) \rightarrow 0
$$

for any measurable $B \subset \mathbb{T}$ (see King [11], Lemma 3.4). It follows that for almost all $\beta \in \mathbb{T}$ there exist infinitely many $n$ such that $\beta \in \Xi_{n}$.

Applying this fact for subsequences of the towers

$$
\left\{\left(T^{j}\left[v_{i}\left\|\alpha q_{n-1}\right\|, w_{i}\left\|\alpha q_{n-1}\right\|\right)\right)_{\tau q_{n}<j<q_{n}}\right\}_{n \in \mathbb{N}}
$$

successively for $i=1, \ldots, k$, we conclude that for $\lambda^{k}$-a.e. $\left(\beta_{1}, \ldots, \beta_{k}\right) \in \mathbb{T}^{k}$ there exist sequences $\left\{n_{j}\right\}_{j \in \mathbb{N}},\left\{t_{i}^{(j)}\right\}_{j \in \mathbb{N}}, i=1, \ldots, k$, of natural numbers such that $\tau q_{n_{j}}<t_{i}^{(j)}<q_{n_{j}}$ and

$$
\begin{aligned}
\beta_{i} \in T^{t_{i}^{(j)}}\left[v_{i}\left\|\alpha q_{n_{j}-1}\right\|, w_{i}\right. & \left.\left\|\alpha q_{n_{j}-1}\right\|\right) \\
& =\left[v_{i}\left\|\alpha q_{n_{j}-1}\right\|+t_{i}^{(j)} \alpha, w_{i}\left\|\alpha q_{n_{j}-1}\right\|+t_{i}^{(j)} \alpha\right) .
\end{aligned}
$$

We can assume that $\left(\left\{q_{n_{j}} \beta_{1}\right\}, \ldots,\left\{q_{n_{j}} \beta_{k}\right\}\right) \rightarrow\left(\gamma_{1}, \ldots, \gamma_{k}\right) \in \mathbb{T}$. Then

$$
\left\{q_{n_{j}} \beta_{i}\right\} \in\left[v_{i} q_{n_{j}}\left\|q_{n_{j}-1} \alpha\right\|+t_{i}^{(j)}\left\|q_{n_{j}} \alpha\right\|, w_{i} q_{n_{j}}\left\|q_{n_{j}-1} \alpha\right\|+t_{i}^{(j)}\left\|q_{n_{j_{l}}} \alpha\right\|\right) .
$$

Since

$$
t_{i}^{(j)}\left\|q_{n_{j}} \alpha\right\| \leq q_{n_{j}}\left\|q_{n_{j}} \alpha\right\| \rightarrow 0 \quad \text { and } \quad q_{n_{j}}\left\|q_{n_{j}-1} \alpha\right\| \rightarrow 1,
$$

as $j \rightarrow \infty$, we have $v_{i} \leq \gamma_{i} \leq w_{i}$ for $i=1, \ldots, k$ and finally $\left(\gamma_{1}, \ldots, \gamma_{k}\right)$ $\in W$.

Let $\Gamma \subset \mathbb{T}^{k}$ denote the set of all $\left(\gamma_{1}, \ldots, \gamma_{k}\right) \in \mathbb{T}^{k}$ such that

$$
\forall_{m_{1}, \ldots, m_{k} \in\{0, \pm 1, \pm 2\}} m_{1} \gamma_{1}+\ldots+m_{k} \gamma_{k} \in \mathbb{Z} \Rightarrow m_{1}, \ldots, m_{k}=0 .
$$

Since the set $\Gamma$ is open and dense, we can choose a cube $W=\prod_{i=1}^{k}\left[v_{i}, w_{i}\right]$ 
(with $0<w_{i}<v_{i+1}<1$ for $i=1, \ldots, k-1$ ) such that $W \subset \Gamma$ and $\lambda^{k}(W)>0$. Fix $1 / 2<\tau<1$. Let $B^{\prime}$ denote the set of all $\left(\beta_{1}, \ldots, \beta_{k}\right) \in \mathbb{T}^{k}$ such that there exists a subsequence $\left\{q_{n_{j}}\right\}_{j \in \mathbb{N}}$ such that

$$
\lim _{j \rightarrow \infty} q_{n_{j}}\left\|q_{n_{j}} \alpha\right\|=0, \quad \lim _{j \rightarrow \infty}\left(\left\{q_{n_{j}} \beta_{1}\right\}, \ldots,\left\{q_{n_{j}} \beta_{k}\right\}\right)=\left(\gamma_{1}, \ldots, \gamma_{k}\right) \in W
$$

and

$$
\beta_{1}, \ldots, \beta_{k} \in \bigcup_{\tau q_{n_{j}}<t<q_{n_{j}}} T^{t}\left[0,\left\|q_{n_{j}-1} \alpha\right\|\right)
$$

for any natural $j$. Then $0=\gamma_{0}<\gamma_{1}<\ldots<\gamma_{k}<\gamma_{k+1}=1$. By Lemma 2.1, $\lambda^{k}\left(B^{\prime}\right)=1$. Define $B_{k}=B^{\prime} \cap \mathbb{T}_{+}^{k}$.

3. Proof of the Main Theorem. For given $f: \mathbb{T} \rightarrow \mathbb{R}$ and $q \in \mathbb{N}$ let

$$
f^{(q)}(x)=f(x)+f(x+\alpha)+\ldots+f(x+(q-1) \alpha) .
$$

Proof of Theorem 1.1. Let $\varphi: \mathbb{T} \rightarrow \mathbb{T}$ be a PAC cocycle and let $0=\beta_{0}<$ $\beta_{1}<\ldots<\beta_{k}<\beta_{k+1}=1$ be all of the points of discontinuity of $\varphi$. Assume that $S(\varphi)=0, \varphi$ has at least one irrational jump and $\left(\beta_{1}, \ldots, \beta_{k}\right) \in B_{k}$. Choose a PAC function $\widetilde{\varphi}: \mathbb{T} \rightarrow \mathbb{R}$ such that $\varphi(x)=e^{2 \pi i \widetilde{\varphi}(x)}$ and $0=$ $\beta_{0}<\beta_{1}<\ldots<\beta_{k}<\beta_{k+1}=1$ are all of the points of discontinuity of $\widetilde{\varphi}$. Let $\left\{q_{n}\right\}_{n \in \mathbb{N}}$ be a subsequence of denominators of $\alpha$ with the properties of Lemma 2.1.

As will be shown in Lemma 3.2 (see $\S 3.2$ ), for all $m \in \mathbb{Z}$ and $r \in \mathbb{N}$ there exists $\delta_{r}^{(m)} \in \mathbb{C}$ such that

$$
\lim _{n \rightarrow \infty} \int_{\mathbb{T}} e^{2 \pi i m \widetilde{\varphi}^{\left(r q_{n}\right)}(x)} d x=\delta_{r}^{(m)} .
$$

This leads to the following statement: each unitary operator $U_{\varphi}^{(m)}$ is $\delta_{r}^{(m)}$ weakly mixing along $\left\{r q_{n}\right\}_{n \in \mathbb{N}}$, by Proposition 2 . Moreover, it will be proved in Lemma 3.3 (see $\S 3.2$ ) that for every $m \in \mathbb{Z} \backslash\{0\}$ there exists $r \in \mathbb{N}$ such that $0<\left|\delta_{r}^{(m)}\right|<1$ and for all distinct $m_{1}, m_{2} \in \mathbb{Z} \backslash\{0\}$, there exists $r \in \mathbb{N}$ such that $\delta_{r}^{\left(m_{1}\right)} \neq \delta_{r}^{\left(m_{2}\right)}$. It follows that the maximal spectral types of the operators $U_{\varphi}^{(m)}$ (for $m \neq 0$ ) are continuous singular and they are mutually singular, by Proposition 1 . The simplicity of the spectrum of $U_{\varphi}^{(m)}$ will be proved in Lemma 3.1 (see §3.1).

Hence each of the operators $U_{T_{\varphi}}: H_{m} \rightarrow H_{m}$ for $m \neq 0$ has simple singular continuous spectrum and their maximal spectral types are pairwise orthogonal. It follows that $T_{\varphi}$ has simple singular continuous spectrum on $H_{0}^{\perp}$. 
3.1. Simplicity of spectrum. Let $V_{g}: L^{2}(\mathbb{T}, \lambda) \rightarrow L^{2}(\mathbb{T}, \lambda)$ be the unitary operator given by

$$
V_{g} f\left(e^{2 \pi i x}\right)=e^{2 \pi i g(x)} f\left(T e^{2 \pi i x}\right),
$$

where $g: \mathbb{T} \rightarrow \mathbb{R}$ is a measurable function. We need the following:

Lemma 3.1. Let $g: \mathbb{T} \rightarrow \mathbb{R}$ be a PAC function with $S(g)=0$. Let $0=\beta_{0}<\beta_{1}<\ldots<\beta_{k}<\beta_{k+1}=1$ be all of the points of discontinuity of $g$. If $\left(\beta_{1}, \ldots, \beta_{k}\right) \in B_{k}$, then $V_{g}$ has simple spectrum.

To prove this lemma we apply the following proposition proved in [5].

Proposition 3. Let $\left\{\Xi_{n}\right\}_{n \in \mathbb{N}}$ be a sequence of towers for the rotation T. Let $C_{n}$ denote the base of $\Xi_{n}$. Suppose that $h_{n}=\operatorname{height}\left(\Xi_{n}\right) \rightarrow \infty$ and $\lambda\left(\bigcup_{j=0}^{h_{n}-1} T^{j} C_{n}\right) \rightarrow \nu$. If there exists $c<\nu$ such that for any $f \in L^{2}(\mathbb{T}, \lambda)$ with $\|f\|_{L^{2}}=1$ we have

$$
\limsup _{n \rightarrow \infty} 2 \pi \sum_{j=0}^{h_{n}-1} \int_{T^{j} C_{n}}|f|^{2} d \lambda \iint_{C_{n}^{2}}\left|g^{(j)}(x)-g^{(j)}(y)\right| \frac{d x d y}{\lambda\left(C_{n}\right)^{2}} \leq c,
$$

then the maximal spectral multiplicity of $V_{g}$ is at most $1 /(\nu-c)$.

Proof of Lemma 3.1. Since $\left(\beta_{1}, \ldots, \beta_{k}\right) \in B_{k}$, we can choose a subsequence $\left\{q_{n}\right\}_{n \in \mathbb{N}}$ of denominators of $\alpha$ with the properties of Lemma 2.1, i.e.

$$
\lim _{n \rightarrow \infty} q_{n}\left\|q_{n} \alpha\right\|=0 \quad \text { and } \quad \beta_{1}, \ldots, \beta_{k} \in \bigcup_{\tau q_{n}<t<q_{n}} T^{t}\left[0,\left\|q_{n-1} \alpha\right\|\right) .
$$

We apply Proposition 3 for the tower $\Xi_{n}=\left(T^{j}\left[0,\left\|q_{n-1} \alpha\right\|\right)\right)_{0 \leq j<\tau q_{n}}$. Then $\lambda\left(\bigcup_{j=0}^{h_{n}-1} T^{j} C_{n}\right) \rightarrow \tau$. Represent $g$ as the sum of an absolutely continuous function $g_{1}: \mathbb{T} \rightarrow \mathbb{R}$ and a piecewise constant $g_{2}: \mathbb{T} \rightarrow \mathbb{R}$. From (2), the function $g_{2}^{(j)}$ is constant on $C_{n}$ for $0 \leq j<\tau q_{n}$. Therefore,

$$
\begin{aligned}
\sum_{0 \leq j<\tau q_{n}} \int_{T^{j} C_{n}}|f|^{2} d \lambda & \iint_{C_{n}^{2}}\left|g^{(j)}(x)-g^{(j)}(y)\right| \frac{d x d y}{\lambda\left(C_{n}\right)^{2}} \\
& =\sum_{0 \leq j<\tau q_{n}} \int_{T^{j} C_{n}}|f|^{2} d \lambda \iint_{C_{n}^{2}}\left|g_{1}^{(j)}(x)-g_{1}^{(j)}(y)\right| \frac{d x d y}{\lambda\left(C_{n}\right)^{2}} .
\end{aligned}
$$

Applying Lemma 4.1 of [5], we can assert that for any $\varepsilon>0$ there exists a subsequence $\left\{\Xi_{n_{l}}\right\}_{l \in \mathbb{N}}$ such that

$$
\limsup _{j \rightarrow \infty} 2 \pi \sum_{0 \leq j<\tau q_{n_{l}}} \int_{T^{j} C_{n_{l}}}|f|^{2} d \lambda \iint_{C_{n_{l}}^{2}}\left|g_{1}^{(j)}(x)-g_{1}^{(j)}(y)\right| \frac{d x d y}{\lambda\left(C_{n_{l}}\right)^{2}} \leq \varepsilon .
$$

Since $\tau>1 / 2$, we can take $\varepsilon<\tau-1 / 2$. Applying Proposition 3 for the 
sequence $\left\{\Xi_{n_{l}}\right\}_{l \in \mathbb{N}}$, we conclude that the maximal spectral multiplicity of $V_{g}$ is at most $1 /(\tau-\varepsilon)<2$.

3.2. $\delta_{r}^{(m)}$-weak mixing

LEMMA 3.2. There exists a real number a such that for all natural $m$ and $r$ we have

$$
\lim _{n \rightarrow \infty} \int_{\mathbb{T}} e^{2 \pi i m \widetilde{\varphi}^{\left(r q_{n}\right)}(x)} d x=\delta_{r}^{(m)}=e^{2 \pi i m r a} \sum_{u=0}^{k}\left(\gamma_{u+1}-\gamma_{u}\right) e^{2 \pi i m r \sum_{i=1}^{u} d_{i}} .
$$

Proof. Set

$$
\phi(x)=\int_{0}^{x} \widetilde{\varphi}(y) d y-\int_{0}^{1} \int_{0}^{z} \widetilde{\varphi}(y) d y d z
$$

and $\psi=\widetilde{\varphi}-\phi$. Then $\phi: \mathbb{T} \rightarrow \mathbb{R}$ is absolutely continuous with zero integral. Moreover $\psi: \mathbb{T} \rightarrow \mathbb{R}$ is constant on each interval $\left(\beta_{i}, \beta_{i+1}\right)$ and $\psi_{-}\left(\beta_{i}\right)-$ $\psi_{+}\left(\beta_{i}\right)=\widetilde{\varphi}_{-}\left(\beta_{i}\right)-\widetilde{\varphi}_{+}\left(\beta_{i}\right)=d_{i}$ for $i=0, \ldots, k$. Of course, we can assume that $\widetilde{\varphi}$ is right continuous. Then

$$
\psi=\psi(0)+\sum_{i=1}^{k+1} d_{i} \mathbf{1}_{\left[\beta_{i}, 1\right)},
$$

where $d_{k+1}=d_{0}$. Since $\phi^{\left(r q_{n}\right)}$ converges uniformly to 0 (see for instance [7], p. 189), and $\widetilde{\varphi}^{\left(r q_{n}\right)}=\phi^{\left(r q_{n}\right)}+\psi^{\left(r q_{n}\right)}$, we see that it suffices to find the limit of the sequence

$$
\int_{\mathbb{T}} e^{2 \pi i m \psi^{\left(r q_{n}\right)}(x)} d x
$$

Since for any $a, b, x \in \mathbb{T}$,

$$
\mathbf{1}_{[b, 1)}(x+a)-\mathbf{1}_{[b, 1)}(a)=\mathbf{1}_{[b-a, 1)}(x)-\mathbf{1}_{[1-a, 1)}(x)
$$

we have

$$
\begin{aligned}
\psi(x+a)-\psi(a) & =\sum_{i=1}^{k+1} d_{i}\left(\mathbf{1}_{\left[\beta_{i}, 1\right)}(x+a)-\mathbf{1}_{\left[\beta_{i}, 1\right)}(x)\right) \\
& =\sum_{i=1}^{k+1} d_{i}\left(\mathbf{1}_{\left[\beta_{i}-a, 1\right)}(x)-\mathbf{1}_{[1-a, 1)}(x)\right) \\
& =\sum_{i=1}^{k+1} d_{i} \mathbf{1}_{\left[\beta_{i}-a, 1\right)}(x) .
\end{aligned}
$$

Therefore for any $r, q \in \mathbb{N}$ we have

$$
\psi^{(r q)}=\psi^{(r q)}(0)+\sum_{h=0}^{q-1} \sum_{s=0}^{r-1} \sum_{i=1}^{k+1} d_{i} \mathbf{1}_{\left[\beta_{i}-(s q+h) \alpha, 1\right)} .
$$


Let $\varrho_{r, q}: \mathbb{T} \rightarrow \mathbb{R}$ be defined by

$$
\varrho_{r, q}=\psi^{(r q)}(0)+r \sum_{j=0}^{q-1} \sum_{i=1}^{k+1} d_{i} \mathbf{1}_{\left[\left(j+\gamma_{i}\right) / q, 1\right)} .
$$

For given $1 \leq i \leq k+1$ and $0 \leq j<q_{n}$ let $h_{i}^{(j)}$ be the unique integer with $0 \leq h_{i}^{(j)}<q_{n}$ such that

$$
h_{i}^{(j)} p_{n}+j=\left[q_{n} \beta_{i}\right] \bmod q_{n} .
$$

Then

$$
\begin{aligned}
\beta_{i}-h_{i}^{(j)} \alpha & =\frac{\left[q_{n} \beta_{i}\right]}{q_{n}}+\frac{\left\{q_{n} \beta_{i}\right\}}{q_{n}}-h_{i}^{(j)} \frac{p_{n}}{q_{n}}-h_{i}^{(j)} \frac{\left\|q_{n} \alpha\right\|}{q_{n}} \\
& =\frac{j}{q_{n}}+\frac{1}{q_{n}}\left(\left\{q_{n} \beta_{i}\right\}-h_{i}^{(j)}\left\|q_{n} \alpha\right\|\right) .
\end{aligned}
$$

Therefore

$$
\psi^{\left(r q_{n}\right)}-\varrho_{r, q_{n}}=\sum_{j=0}^{q_{n}-1} \sum_{s=0}^{r-1} \sum_{i=1}^{k+1} d_{i}\left(\mathbf{1}_{\left[\beta_{i}-\left(s q_{n}+h_{i}^{(j)}\right) \alpha, 1\right)}-\mathbf{1}_{\left[\left(j+\gamma_{i}\right) / q_{n}, 1\right)}\right),
$$

and

$$
\left\|\psi^{\left(r q_{n}\right)}-\varrho_{r, q_{n}}\right\|_{L^{1}} \leq D \sum_{j=0}^{q_{n}-1} \sum_{s=0}^{r-1} \sum_{i=1}^{k+1}\left|\beta_{i}-\left(s q_{n}+h_{i}^{(j)}\right) \alpha-\left(j+\gamma_{i}\right) / q_{n}\right|,
$$

where $D=\max _{i=1, \ldots, k+1}\left|d_{i}\right|$. We conclude from (4) that

$$
\begin{aligned}
\left\|\psi^{\left(r q_{n}\right)}-\varrho_{r, q_{n}}\right\|_{L^{1}} & \leq D \sum_{j=0}^{q_{n}-1} \sum_{s=0}^{r-1} \sum_{i=1}^{k+1}\left|\frac{\left\{q_{n} \beta_{i}\right\}-\gamma_{i}}{q_{n}}-\left(s+\frac{h_{i}^{(j)}}{q_{n}}\right)\left\|q_{n} \alpha\right\|\right| \\
& \leq D r \sum_{i=1}^{k}\left|\left\{q_{n} \beta_{i}\right\}-\gamma_{i}\right|+D k r^{2} q_{n}\left\|q_{n} \alpha\right\|,
\end{aligned}
$$

and hence that

$$
\lim _{n \rightarrow \infty}\left\|\psi^{\left(r q_{n}\right)}-\varrho_{r, q_{n}}\right\|_{L^{1}}=0
$$

On the other hand

$$
\begin{aligned}
\varrho_{r, q} & =\psi^{(r q)}(0)+r \sum_{j=0}^{q-1} \sum_{i=1}^{k+1} d_{i}\left(\sum_{u=i}^{k} \mathbf{1}_{\left[\left(j+\gamma_{u}\right) / q,\left(j+\gamma_{u+1}\right) / q\right)}+\mathbf{1}_{[(j+1) / q, 1)}\right) \\
& =\psi^{(r q)}(0)+r \sum_{j=0}^{q-1} \sum_{u=1}^{k} \sum_{i=1}^{u} d_{i} \mathbf{1}_{\left[\left(j+\gamma_{u}\right) / q,\left(j+\gamma_{u+1}\right) / q\right)}
\end{aligned}
$$


and consequently

$$
\begin{aligned}
\int_{\mathbb{T}} e^{2 \pi i m \varrho_{r, q}(x)} d x & =e^{2 \pi i m \psi^{(r q)}(0)} \sum_{j=0}^{q-1} \sum_{u=1}^{k} \frac{1}{q}\left(\gamma_{u+1}-\gamma_{u}\right) e^{2 \pi i m r \sum_{i=1}^{u} d_{i}} \\
& =e^{2 \pi i m \psi^{(r q)}(0)} \sum_{u=0}^{k}\left(\gamma_{u+1}-\gamma_{u}\right) e^{2 \pi i m r \sum_{i=1}^{u} d_{i}} .
\end{aligned}
$$

Without loss of generality we can assume that

$$
\lim _{n \rightarrow \infty} e^{2 \pi i \psi^{\left(q_{n}\right)}(0)}=e^{2 \pi i a} .
$$

Then

$$
\lim _{n \rightarrow \infty} e^{2 \pi i \psi^{\left(r q_{n}\right)}(0)}=e^{2 \pi i r a} .
$$

Indeed, since $\left\{q_{n} \beta_{i}\right\} \rightarrow \gamma_{i}>\gamma_{1}>0$ and $q_{n}\left\|q_{n} \alpha\right\| \rightarrow 0$, we have

$$
q_{n}\left\|q_{n} \alpha\right\|<\min _{i=1, \ldots, k}\left\{q_{n} \beta_{i}\right\} / r
$$

for sufficiently large $n$. Then for any $i=1, \ldots, k, j=0, \ldots, q_{n}$, we have

$$
(r-1)\left\|q_{n} \alpha\right\|<\frac{\left\{q_{n} \beta_{i}\right\}}{q_{n}}-\left\|q_{n} \alpha\right\| \leq \frac{\left\{q_{n} \beta_{i}\right\}}{q_{n}}+\frac{j}{q_{n}}-\frac{h_{i}^{(j)}\left\|q_{n} \alpha\right\|}{q_{n}}=\beta_{i}-h_{i}^{(j)} \alpha .
$$

It follows that $\psi^{\left(q_{n}\right)}(0)=\psi^{\left(q_{n}\right)}\left(q_{n} \alpha\right)=\ldots=\psi^{\left(q_{n}\right)}\left((r-1) q_{n} \alpha\right)$, by $(3)$. Since

$$
\psi^{\left(r q_{n}\right)}(0)=\psi^{\left(q_{n}\right)}(0)+\psi^{\left(q_{n}\right)}\left(q_{n} \alpha\right)+\ldots+\psi^{\left(q_{n}\right)}\left((r-1) q_{n} \alpha\right),
$$

we have $\psi^{\left(r q_{n}\right)}(0)=r \psi^{\left(q_{n}\right)}(0)$. From (5)-(7), we obtain

$$
\lim _{n \rightarrow \infty} \int_{\mathbb{T}} e^{2 \pi i m \psi^{\left(r q_{n}\right)}(x)} d x=e^{2 \pi i m r a} \sum_{u=0}^{k}\left(\gamma_{u+1}-\gamma_{u}\right) e^{2 \pi i m r \sum_{i=1}^{u} d_{i}} .
$$

Lemma 3.3. For every $m \in \mathbb{Z} \backslash\{0\}$ there exists $r \in \mathbb{N}$ such that $0<$ $\left|\delta_{r}^{(m)}\right|<1$ and for all distinct $m_{1}, m_{2} \in \mathbb{Z} \backslash\{0\}$ there exists $r \in \mathbb{N}$ such that $\delta_{r}^{\left(m_{1}\right)} \neq \delta_{r}^{\left(m_{2}\right)}$.

Proof. Let $G \subset \mathbb{T}$ be the subgroup generated by $1, e^{2 \pi i d_{1}}, e^{2 \pi i\left(d_{1}+d_{2}\right)}$, $\ldots, e^{2 \pi i\left(d_{1}+\ldots+d_{k}\right)}$. Let us decompose

$$
G=e^{2 \pi i \alpha_{1} \mathbb{Z}} \oplus \ldots \oplus e^{2 \pi i \alpha_{g} \mathbb{Z}} \oplus G_{1},
$$

where $G_{1}$ is a finite group $\left(c=\operatorname{card} G_{1}\right)$ and $\alpha_{1}, \ldots, \alpha_{g}, 1$ are independent over $\mathbb{Q}$. As some of $d_{j}$ is irrational, we have $g=\operatorname{rank}(G)>0$. Let $\left[a_{i j}\right]_{i=1, \ldots, g ; j=1, \ldots, k}$ be an integer matrix such that

$$
e^{2 \pi i c\left(d_{1}+\ldots+d_{j}\right)}=e^{2 \pi i\left(a_{j 1} \alpha_{1}+\ldots+a_{j g} \alpha_{g}\right)}
$$


for $j=1, \ldots, k$. Define $\omega_{j}=e^{2 \pi i \alpha_{j}}$ for $j=1, \ldots, g$ and $\omega_{0}=e^{2 \pi i c a}$. Set $\lambda_{j}=\gamma_{j+1}-\gamma_{j}$ for $j=0, \ldots, k$. Then $\lambda_{0}, \ldots, \lambda_{k}>0$ and $\lambda_{0}+\ldots+\lambda_{k}=1$. Let $Q$ denote the trigonometric polynomial on $\mathbb{T}^{g}$ given by

$$
Q\left(z_{1}, \ldots, z_{g}\right)=\lambda_{0}+\lambda_{1} z_{1}^{a_{11}} \ldots z_{g}^{a_{1 g}}+\ldots+\lambda_{k} z_{1}^{a_{k 1}} \ldots z_{g}^{a_{k g}} .
$$

Then

$$
\delta_{c r}^{(m)}=\omega_{0}^{m r} Q\left(\omega_{1}^{m r}, \omega_{2}^{m r}, \ldots, \omega_{g}^{m r}\right) .
$$

Since some of $d_{1}+\ldots+d_{j}$ for $j=1, \ldots, k$ are irrational, it is easy to see that $\left|\delta_{c r}^{(m)}\right|<1$ for all $m, r \neq 0$.

We now show that for any $m \neq 0$ there exists $r \in \mathbb{N}$ such that

$$
0<\left|Q\left(\omega_{1}^{m r}, \ldots, \omega_{g}^{m r}\right)\right|<1 .
$$

Suppose that for all $r \in \mathbb{N}$, we have $Q\left(\omega_{1}^{m r}, \ldots, \omega_{g}^{m r}\right)=0$. Since $\alpha_{1}, \ldots, \alpha_{g}, 1$ are independent over $\mathbb{Q}, Q\left(z_{1}, \ldots, z_{g}\right)=0$ for any $\left(z_{1}, \ldots, z_{g}\right) \in \mathbb{T}^{g}$. Hence $0=Q(1, \ldots, 1)=1$, a contradiction.

Let us show that if $|m| \neq\left|m^{\prime}\right|, m, m^{\prime} \neq 0$, then there exists $r \in \mathbb{N}$ such that

$$
\left|Q\left(\omega_{1}^{m r}, \ldots, \omega_{g}^{m r}\right)\right| \neq\left|Q\left(\omega_{1}^{m^{\prime} r}, \ldots, \omega_{g}^{m^{\prime} r}\right)\right| .
$$

Suppose, contrary to our claim, that equality occurs in (8) for any $r \in \mathbb{N}$. Then

$$
\left|Q\left(z_{1}^{m}, \ldots, z_{g}^{m}\right)\right|=\left|Q\left(z_{1}^{m^{\prime}}, \ldots, z_{g}^{m^{\prime}}\right)\right| \quad \text { for any }\left(z_{1}, \ldots, z_{g}\right) \in \mathbb{T}^{g} .
$$

Let $P$ denote the trigonometric polynomial on $\mathbb{T}$ given by

$$
P(z)=\left|Q\left(z^{m}, 1, \ldots, 1\right)\right|^{2}=\left|Q\left(z^{m^{\prime}}, 1, \ldots, 1\right)\right|^{2} .
$$

Since

$$
\max _{i, j=0, \ldots, k}\left|m\left(a_{i 1}-a_{j 1}\right)\right|=\max _{i, j=0, \ldots, k}\left|m^{\prime}\left(a_{i 1}-a_{j 1}\right)\right|=\operatorname{deg} P>0,
$$

where $a_{01}=0$, we obtain $|m|=\left|m^{\prime}\right|$, a contradiction.

Let us show that for any $m \neq 0$ there exists $r \in \mathbb{N}$ such that

$$
\omega_{0}^{m r} Q\left(\omega_{1}^{m r}, \ldots, \omega_{g}^{m r}\right) \neq \omega_{0}^{-m r} Q\left(\omega_{1}^{-m r}, \ldots, \omega_{g}^{-m r}\right) .
$$

Suppose that equality occurs in (9) for all $r \in \mathbb{N}$. Then

$$
\omega_{0}^{m r} Q\left(\omega_{1}^{m r}, \ldots, \omega_{g}^{m r}\right) \in \mathbb{R} \quad \text { for all } r \in \mathbb{Z} .
$$

Set $G_{0}=\left\{\left(\omega_{1}^{r}, \ldots, \omega_{g}^{r}\right): r \in \mathbb{Z}\right\}$. Let $F: G_{0} \rightarrow \mathbb{T}$ be the group homomorphism given by 


$$
F\left(\omega_{1}^{r}, \ldots, \omega_{g}^{r}\right)=\omega_{0}^{2 m r}=\frac{Q\left(\omega_{1}^{-m r}, \ldots, \omega_{g}^{-m r}\right)}{Q\left(\omega_{1}^{m r}, \ldots, \omega_{g}^{m r}\right)} .
$$

Then $\left(\omega_{1}^{r_{n}}, \ldots, \omega_{g}^{r_{n}}\right) \rightarrow(1, \ldots, 1)$ implies

$$
F\left(\omega_{1}^{r_{n}}, \ldots, \omega_{g}^{r_{n}}\right)=\frac{Q\left(\omega_{1}^{-m r_{n}}, \ldots, \omega_{g}^{-m r_{n}}\right)}{Q\left(\omega_{1}^{m r_{n}}, \ldots, \omega_{g}^{m r_{n}}\right)} \rightarrow \frac{Q(1, \ldots, 1)}{Q(1, \ldots, 1)}=F(1, \ldots, 1) .
$$

Since $F$ is a continuous group homomorphism and $\bar{G}_{0}=\mathbb{T}^{g}$, there exists a continuous group homomorphism $\bar{F}: \mathbb{T}^{g} \rightarrow \mathbb{T}$ such that $\left.\bar{F}\right|_{G_{0}}=F$ and

$$
\bar{F}\left(z_{1}, \ldots, z_{g}\right)=z_{1}^{c_{1}} \ldots z_{g}^{c_{g}},
$$

where $c_{1}, \ldots, c_{g} \in \mathbb{Z}$. Therefore

$$
\omega_{0}^{2 m}=F\left(\omega_{1}, \ldots, \omega_{g}\right)=\omega_{1}^{c_{1}} \ldots \omega_{g}^{c_{g}}
$$

and consequently

$$
\omega_{1}^{c_{1} r} \ldots \omega_{g}^{c_{g} r} Q\left(\omega_{1}^{2 m r}, \ldots, \omega_{g}^{2 m r}\right) \in \mathbb{R}
$$

for all $r \in \mathbb{Z}$. It follows that the trigonometric polynomial

$$
z_{1}^{c_{1}} \ldots z_{g}^{c_{g}} Q\left(z_{1}^{2 m}, \ldots, z_{g}^{2 m}\right)
$$

has only real values. Hence there exist $m_{0}, \ldots, m_{k} \in\{0,1,-1\}$ such that $\sum_{j=0}^{k} m_{j} \lambda_{j}=0$ and there exist $j_{1}, j_{2}$ such that $m_{j_{1}}=1$ and $m_{j_{2}}=-1$, contrary to $\left(\gamma_{1}, \ldots, \gamma_{k}\right) \in \Gamma$.

\section{References}

[1] H. Anzai, Ergodic skew product transformations on the torus, Osaka Math. J. 3 (1951), 83-99.

[2] G. H. Choe, Spectral types of skewed irrational rotations, Comm. Korean Math. Soc. 8 (1993), 655-668.

[3] P. Gabriel, M. Lemańczyk et P. Liardet, Ensemble d'invariants pour les produits croisés de Anzai, Mém. Soc. Math. France 47 (1991).

[4] G. R. Goodson, J. Kwiatkowski, M. Lemańczyk and P. Liardet, On the multiplicity function of ergodic group extensions of rotations, Studia Math. 102 (1992), 157-174.

[5] M. Guenais, Une majoration de la multiplicité spectrale d'opérateurs associés à des cocycles réguliers, Israel J. Math. 105 (1998), 263-283.

[6] H. Helson, Cocycles on the circle, J. Operator Theory 16 (1986), 189-199.

[7] M. Herman, Sur la conjugaison différentiable des difféomorphismes du cercle à des rotations, Publ. Mat. IHES 49 (1979), 5-234.

[8] A. Iwanik, Generic smooth cocycles of degree zero over irrational rotation, Studia Math. 115 (1995), 241-250.

[9] A. Iwanik, M. Lemańczyk and C. Mauduit, Piecewise absolutely continuous cocycles over irrational rotations, J. London Math. Soc. 59 (1999), 171-187. 
[10] A. Iwanik, M. Lemańczyk and D. Rudolph, Absolutely continuous cocycles over irrational rotations, Israel J. Math. 83 (1993), 73-95.

[11] J. L. King, Joining-rank and the structure of finite rank mixing transformations, J. Anal. Math. 51 (1988), 182-227.

Faculty of Mathematics and Computer Science

Nicholas Copernicus University

Chopina 12/18

87-100 Toruń, Poland

E-mail: fraczek@mat.uni.torun.pl

Received October 5, 1998

Revised version December 11, 2000 